Disclosure of Interests: Karishma Shah: None declared, Garrett Bullock: None declared, Alan Silman: None declared, Dominic Furniss: None declared, Nigel Arden Consultant of: Receives personal fees from Pfizer/Lily for consultancy outside the scope of this work, Grant/research support from: Receives grant from Merck outside the scope of this work, Gary Collins: None declared DOI: 10.1136/annrheumdis-2021-eular.1126

\section{POS0126 EARLY STAGE HAND OSTEOARTHRITIS IN PATIENTS WITH THE EHLERS-DANLOS SYNDROME: AN EXPLORATIVE STUDY}

L. Rombaut ${ }^{1}$, K. De Baere ${ }^{2}$, E. Debacker ${ }^{2}$, L. Decavele ${ }^{2}$, F. Malfait ${ }^{1,3}$, R. Wittoek ${ }^{4,5},{ }^{1}$ Ghent University Hospital, Center for Medical Genetics, Gent, Belgium; ${ }^{2}$ Ghent University, Rehabilitation Sciences, Ghent, Belgium; ${ }^{3}$ Ghent University, Department of Biomolecular Medicine, Ghent, Belgium; ${ }^{4}$ Ghent University, Internal Medicine and Pediatrics, Ghent, Belgium; ${ }^{5}$ Ghent University Hospital, Department of Rheumatology, Gent, Belgium

Background: The Ehlers-Danlos Syndromes (EDS) are a group of rare heritable connective tissue disorders caused by various defects in the biosynthesis or secretion of fibrillar collagens. The three main clinical features of EDS are joint hypermobility, skin fragility and general soft tissue fragility. 13 clinical subtypes of EDS are recognized, of which the hypermobile type (hEDS) and classical type (cEDS) are the most prevalent. It has been hypothesized that the (micro-)trauma in the joint due to typical subluxations and dislocations, make EDS patients prone to developing osteoarthritis $(\mathrm{OA})$ in early stage. Conversely, it has been mentioned that joint hypermobility provides a larger joint surface area and prevents OA. Abnormal biomechanical loading has been identified as a risk factor for the development of OA in the wrist and hand. However, no studies have yet been performed in EDS patients.

Objectives: The primary aim was to investigate the presence of any degenerative features for hand OA, and if this differs between cEDS and hEDS patients. The second aim was to evaluate hand function and pain related to $O A$ signs in EDS patients.

Methods: cEDS and hEDS patients between 35 and 50 years old were invited to participate. CEDS diagnosis was genetically confirmed and hEDS diagnosis was performed according the clinical $2017 \mathrm{hEDS}$ criteria. Exclusion criteria were a body mass index $\geq 35$, not being able to stand straight up for five minutes, suffering from an auto-immune disease or rheumatological condition, or pregnancy. Conventional X-rays of both hands were performed and scored independently by three assessors according to Kallman ${ }^{1}$. Presence of osteophytes (0-3), joint space narrowing $(0-3)$, malalignment $\left(>15^{\circ}\right)(0 / 1)$, erosions $(0 / 1)$, subchondral sclerosis $(0 / 1)$, and subchondral cysts $(0 / 1)$ were scored in all interphalangeal (distal and proximal), metacarpophalangeal and thumb base joints of both hands ${ }^{1}$. We defined early hand $O A$ as minimally three features $(\geq 1)$ were present. Several clinical assessments were made, e.g. tenderness, bony swelling and soft tissue swelling. The Michigan Hand Outcomes Questionnaire (MHOQ) and Australian/Canadian Osteoarthritis Hand Index (AUSCAN) questionnaires were completed.

Results: In total, 31 patients (mean age $41 \pm 5.6$ years, 13 men and 18 women) diagnosed with EDS participated, of whom 19 with CEDS and with 12 hEDS. In total, 927 joints were assessed. Level of agreement of radiographic assessments was very high (>98\%). Early hand OA was found in more than $40 \%$ of the EDS patients, with a significant higher frequency in cEDS patients compared to hEDS patients $(58 \%$ vs. $17 \%)(p=0.032)$. Joint space narrowing was most frequently present and significantly more in the cEDS patients compared to hEDS patients $(79 \%$ vs. $21 \%)(p=0.003)$. However, radiographic changes were found in only $10 \%$ of all finger joints. Of all fingers, thumb joints were most affected. Regarding the clinical features of hand OA, all patients showed deformity in one or more finger joints, most frequently at the thumb, especially the IP joint (both hyperflexion and hyperextension). Tenderness and bony swelling was present in $36 \%$ and $45 \%$ of all patients, respectively, whereas soft tissue swelling was less frequently observed ( $10 \%$ of all patients). Here, no significant differences were found between cEDS and hEDS patients. Moderate disability was present (mean (SD) AUSCAN= 45.47 (27.10) and MHQ $=65.97$ (14.21). cEDS showed significant less hand pain $(p=0.03)$, a better hand function $(p=0.03)$ and less disability $(\mathrm{p}=0.026)$ than hEDS.

Conclusion: This explorative study demonstrates that a high number of EDS patients present with minimal degenerative features of hand $\mathrm{OA}$, but in a minority of joints. Patients with cEDS were significantly more affected, but showed a better function compared to hEDS. Possibly, cEDS patients are more susceptible to develop hand OA.

REFERENCES:

[1] Altman R, Gold G. Atlas of individual radiographic features in osteoarthritis, revised. OARSI. 2007;15:A1-A56.

Disclosure of Interests: None declared

DOI: 10.1136/annrheumdis-2021-eular.1351

\section{POS0127 ASSOCIATION BETWEEN HYPERURICEMIA AND OSTEOARTHRITIS}

E. Taskina ${ }^{1}$, E. Strebkova ${ }^{1}$, L. Alekseeva ${ }^{1,2}$, N. Kashevarova ${ }^{1}$, K. Telyshev ${ }^{1}$, E. Sharapova' ${ }^{1}$, S. Anikin ${ }^{1}$, F. Bibulatova ${ }^{1}$, A. Lila ${ }^{2,3} .{ }^{1}$ V. A. Nasonova Research Institute of Rheumatology, Osteoarthritis, Moscow, Russian Federation; ${ }^{2}$ Russian Medical Academy of Continuous Professional Education, Rheumatology, Moscow, Russian Federation; ${ }^{3} \mathrm{~V}$. A. Nasonova Research Institute of Rheumatology, Director, Moscow, Russian Federation

\section{Background:}

Objectives: To evaluate the relationship of hyperuricemia (GU) with the course of knee osteoarthritis (OA) and the components of metabolic syndrome (MS). Methods: The prospective study included 107 women aged 40-75 y.o, with a reliable diagnosis of I-III Kellgren J. - Lawrence J. stage of knee OA (ACR), who signed an informed consent. The average age of patients was $59.1 \pm 8.9$ y.o. (from 38 to 74), the duration of the disease was 9 (4-12) years. The average values of the body mass index (BMI) corresponded to obesity $(30.6 \pm 5.7 \mathrm{~kg} /$ $\mathrm{m} 2$ ), waist circumference (WC) $-93.9 \pm 12.1 \mathrm{~cm}$. An individual card was filled out for each patient, including anthropometric parameters, anamnesis and clinical examination data, an assessment of knee joint pain according to VAS, and concomitant diseases. All patients underwent standard X-ray of the knee joints, DEXA scan of the lumbar spine and total hip, and laboratory examination.

Results: Hyperuricemia was diagnosed in $26.2 \%$ of patients. Patients with GU had a higher BMI and WC $(p<0.05)$. X-ray examination revealed significantly larger sizes of osteophytes $(p=0.02)$ and smaller sizes of the medial space of the knee joint $(p=0.02)$, while the patients of both groups were comparable in age and duration of OA (Table 1). Patients with GU had a statistically significantly higher bone mineral density (BMD) values were observed in L1-L4 and total hip $(\mathrm{p}<0.05)$. MS was almost 2 times more often diagnosed in patients with GU $(83.3 \%$ vs. $49.2 \%, R R=1.71$ $95 \% \mathrm{Cl} 1.21-2.36, \mathrm{p}=0.01)$. Higher levels of CRP, COMP, leptin, triglycerides (TG), creatinine, ALT and AST were determined in patients with GU (Table 1). Spearman rank ordered correlation coefficients between $\mathrm{GU}$ and $\mathrm{BMI}(\mathrm{r}=0.22, \mathrm{p}=0.03)$, WC $(r=0.26, p=0.02)$, MS $(r=0.29, p=0.01)$, and $\mathrm{CHD}(r=0.21, p=0.04)$. There were a correlation between $\mathrm{GU}$ and the stage of knee $\mathrm{OA}(\mathrm{X}$-ray) $(r=0.34, p=0.02)$, the size of medial osteophytes in the femur $(\mathrm{mm})(\mathrm{r}=0.37, \mathrm{p}=0.01)$ and the medial space of the knee joint $(\mathrm{mm})(r=-0.27, p=0.01), B M D$ L1-L4 $(r=0.37, p=0.03)$ and the total hip $(r=0.37, p=0.04)$. There were also significant positive associations with the following laboratory parameters: $\operatorname{CRP}(r=0.27, p=0.007), \operatorname{COMP}(r=0.25, p=0.05)$, leptin $(r=0.29, p=0.03)$, creatinine $(r=0.45, p<0.0001), T G(r=0.44, p=0.0001), A L T(r=0.32$, $p=0.002)$, AST $(r=0.25, p=0.02)$.

Table 1. Comparative characteristics of OA patients with and without GU

\begin{tabular}{lccc}
\hline Parameter & $\begin{array}{c}\text { OA Patients with GU } \\
(\mathrm{n}=28)\end{array}$ & $\begin{array}{c}\text { OA Patients without GU } \\
(\mathrm{n}=79)\end{array}$ & $\mathrm{p}$ \\
\hline Age, y.o. & $61(53-68)$ & $60(54-66)$ & 0.33 \\
Duration OA, years & $10(6-14)$ & $7.5(3-12)$ & 0.06 \\
WC, cm & $99.3 \pm 12.8$ & $92.2 \pm 11.4$ & 0.02 \\
BMI, kg/m & $33.5 \pm 5.6$ & $30 \pm 5.6$ & 0.04 \\
The size of the medial space of joint & $2.3(1.2-3.3)$ & $3.2(2.0-4.3)$ & 0.02 \\
$\quad$ & & & \\
$\quad$ according to X-ray, mm & $5.8(3.7-6.3)$ & $2.9(1.7-4.5)$ & 0.02 \\
The size of the osteophytes of the & & & \\
$\quad$ femur, mm & $1.18(1.12-1.24)$ & $1.0(0.86-1.15)$ & 0.04 \\
BMI L1-L4, g/cm & $1.05(1.0-1.13)$ & $0.9(0.82-0.98)$ & 0.04 \\
BMI total hip, g/cm & $2.8(1.9-6.2)$ & $1.9(1.2-3.6)$ & 0.007 \\
CRP, mg/l & $31.9(27.6-45)$ & $24.2(18.9-38.7)$ & 0.05 \\
COMP, $\mathrm{ng} / \mathrm{ml}$ & $46.2(36.5-72)$ & $30.5(19.5-45.6)$ & 0.03 \\
Leptin, $\mathrm{ng} / \mathrm{ml}$ & $1.97(1.61-2.41)$ & $1.26(0.87-1.63)$ & 0.002 \\
TG, mmol/l & $79(74.9-86)$ & $68(62-74)$ & 0.0001 \\
Creatinine, mmol/l & $26.5(17.2-32.9)$ & $17.9(13.2-21.7)$ & 0.003 \\
ALT, mmol/l & $22.5(18.5-27.8)$ & $18.9(16.8-21.9)$ & 0.02 \\
AST, mmol/l & & &
\end{tabular}

Conclusion: GU may be an aggravating predictor of not only MS-associated diseases such as obesity, dyslipidemia, but also OA. Thus, at high levels of uric acid, more advanced radiological stages of $\mathrm{OA}$, high values of the BMD of the axial skeleton and increased values of CRP, COMP are noted. In this connection, therapeutic interventions of $O A$ in patients with GU should also be aimed at reducing the concentration of uric acid, which may provide a slowdown in the progression of the disease. Further study of this issue is needed.

Disclosure of Interests: None declared

DOI: 10.1136/annrheumdis-2021-eular.3364

\section{POS0128 PHYSICIAN AND PATIENT PERCEPTIONS OF SURGICAL PROCEDURES FOR KNEE OA ACROSS JAPAN, THE US AND 5 EU COUNTRIES: RESULTS OF A REAL-WORLD STUDY}

N. Fukui', P. G. Conaghan ${ }^{2}$, K. Togo ${ }^{3}$, N. Ebata ${ }^{3}$, L. Abraham ${ }^{4}$, J. Jackson ${ }^{5}$, J. Jackson ${ }^{5}$, M. Berry ${ }^{5}$, H. Pandit ${ }^{2}{ }^{1}$ The University of Tokyo Komaba Campus, 
Graduate School of Arts and Sciences, Meguro City, Japan; ${ }^{2}$ University of Leeds, Musculoskeletal Medicine, Leeds, United Kingdom; ${ }^{3}$ Pfizer, Inc., HEOR, Shibuya City, Japan; ${ }^{4}$ Pfizer Ltd, HEOR, Surrey, United Kingdom; ${ }^{5}$ Adelphi Real World, Real World Research, Bollington, United Kingdom

Background: Patients with knee osteoarthritis (OA) who do not achieve adequate pain relief and functional improvement with a combination of non-pharmacologic and pharmacologic therapies are recommended an arthroplasty as an effective option to relieve severe pain and functional limitations. However, some patients are reluctant to undergo surgical interventions, and clinicians may choose to avoid or delay surgery due to safety risks and/or the financial cost. It is of interest to understand if the use and perception of surgery differs between countries, however, few published data exist.

Objectives: To demonstrate how surgery and the use of surgical procedures differs across Japan, United States of America (US) and 5 major European countries (EU5) and to evaluate patient perception towards surgery.

Methods: Data were drawn from the Adelphi OA Disease Specific Programme (2017-18), a point-in-time survey of primary care physicians (PCP), rheumatologists (rheums), orthopaedic surgeons (orthos) and their OA patients. Patients with physician-diagnosed knee OA were included and segmented into two categories: had previous surgery (PS) and never had surgery (NS). A Fisher's exact test was performed on the two groups. Physicians reported on patient demographics; whether patients had undergone surgery; type of surgery; success of surgery; how success was defined; and reasons for wanting to delay surgery. Patients reported their willingness to undergo surgery; reasons for not wanting surgery; how successful their surgery was; and how they defined this success. Results: Physician/patient reported data were available for 302,230 (Japan), 527,283 (US) and 1487,726 (EU5) patients with diagnosed knee OA. Patients were categorised by their physicians as mild (40\% Japan; 34\% US; $24 \%$ EU5), moderate (49\% Japan; $49 \%$ US; $56 \%$ EU5) or severe (9\% Japan; $17 \%$ US; $19 \%$ EU5). Patients in Japan were more likely to be female ( $78 \%$ vs $54 \%$ US; $58 \%$ EU5), older (73 vs 65 US; 66 EU5) and have a lower BMI than patients in the US and EU5. Obesity and diabetes were much less prevalent among patients in Japan. One in ten patients in Japan had undergone a surgery (10\%), far fewer than in the US (22\%) or EU5 (17\%). When surgery was performed, this was more likely to be a total joint replacement (TJR) in Japan, whereas in the EU and US, arthroscopic washout was more commonly performed.

For over half of Japanese patients (56\%), successful surgery was more likely to be defined as having no more pain (vs. $35 \%$ US; $14 \%$ EU5). Improved mobility and a reduction in pain were also commonly reported reasons. Physicians (in each region) were more likely to suggest pain reduction, rather than no pain, and improved mobility as markers of success. Patients in Japan were much more likely to say they would not agree to surgery if recommended by their doctor, or were unsure (84\% vs. $68 \%$ US; $62 \%$ EU5). The main reason for patient reluctance in Japan was fear of surgery, whereas in the US and EU5 the main reason given was that surgery was not needed. This finding was also evident among physicians in Japan, who frequently reported that patient reluctance was a key reason for delaying surgery. Physicians in Japan, do however, report that patient request was one of their main triggers for recommending surgery ( $45 \%$ vs $20 \%$ US; $16 \%$ EU5).

Conclusion: Although surgery can be an effective option for those with OA who have exhausted other treatment options, some patients are reluctant to undergo surgery out of fear, especially in Japan, possibly due to the higher patient age. Physicians aiming to delay surgery were driven by patient reluctance in Japan, whereas cost to patient was a bigger factor in the US and EU5. The higher level of TJR vs. other surgery options among patients in Japan may suggest physicians are looking for higher levels of efficacy.

Disclosure of Interests: Naoshi Fukui Speakers bureau: Pfizer, Consultant of: Pfizer, Philip G Conaghan Speakers bureau: Abbvie, Novartis, Consultant of: AstraZeneca, BMS, Eli Lilly, EMD Serono, Flexion Therapeutics, Galapagos, Gilead, Novartis, Pfizer, Kanae Togo Shareholder of: Pfizer, Employee of: Pfizer, Nozomi Ebata Shareholder of: Pfizer, Employee of: Pfizer, Lucy Abraham Shareholder of: Pfizer, Employee of: Pfizer, James Jackson: None declared, Jessica Jackson: None declared, Mia Berry: None declared, Hemant Pandit Paid instructor for: Bristol Myers Squibb, Consultant of: Johnson and Johnson, Grant/ research support from: GSK

DOI: 10.1136/annrheumdis-2021-eular.2510

\section{POS0129 ASSOCIATION BETWEEN OSTEOARTHRITIS-RELATED SERUM BIOCHEMICAL MARKERS OVER 11 YEARS AND KNEE MRI-BASED IMAGING BIOMARKERS IN MIDDLE-AGED ADULTS}

B. Antony ${ }^{1}$, A. Singh ${ }^{1}$, L. Blizzard ${ }^{1}$, A. Venn ${ }^{1}$, G. Jones ${ }^{1}$, J. Burgess ${ }^{1}$, V. Parameswaran ${ }^{2}$, F. Cicuttini ${ }^{3}$, L. March ${ }^{4}$, C. Ding ${ }^{1} .{ }^{1}$ University of Tasmania, Menzies Institute for Medical Research, Hobart, Australia; ${ }^{2}$ Royal Hobart Hospital, Department of Endocrinology, Hobart, Australia; ${ }^{3}$ Monash University,
Department of Epidemiology and Preventive Medicine, Melbourne, Australia; ${ }^{4}$ University of Sydney, Institute of Bone and Joint Research, Kolling Institute of Medical Research, Sydney, Australia

Background: Serum levels of osteoarthritis (OA)-related cartilage and joint-specific biochemical markers - cartilage oligomeric matrix protein (COMP), matrix metalloproteinase (MMP)-3, and hyaluronan (HA) -are shown to be associated with cartilage degradation, joint tissue degradation, and synovitis in patients with $\mathrm{OA}$. Although these OA-related biochemical markers may initially precede the MRI biomarkers of joint structural changes, such changes detected in MRI can lead to biochemical marker changes later as the condition progresses. However, there is a lack of data on OA-related biochemical markers' association with MRIbased biomarkers in the middle-aged general population.

Objectives: We aimed to describe the associations between OA-related biochemical markers and MRI-based imaging biomarkers in middle-aged adults followed up over 10-13 years.

Methods: Blood samples were collected during the Childhood Determinants of Adult Health (CDAH)-1 study at baseline (year: 2004-06, age: $26-36$ years) and 10-13 year follow-up (CDAH-3; year: 2014-2019, age: 36-49 years). Serum samples from baseline $(n=156)$ and follow-up $(n=167)$ were analyzed for three OA-related biomarkers - namely COMP, MMP-3, and HA- using non isotopic ELISA assay methodology. Knee MRI scans were obtained during the CDAH-knee study (year: 2008-10, age: 30-40 years, $n=313$ ), and MRIs were assessed for cartilage volume, cartilage thickness, subchondral bone area, cartilage defects, and bone marrow lesions (BML). Univariable and multivariable (adjusted for age, sex, and body mass index (BMI)) linear regression and logistic regression were used to describe the association of biochemical marker at CDAH-1 and MRIbased imaging biomarkers at CDAH-knee, and Tobit regression was used to describe the association of MRI-based imaging biomarkers at CDAH-knee and biochemical markers at $\mathrm{CDAH}-3$.

Results: In the multivariable model for the association of biochemical marker with MRI-based imaging biomarkers (assessed after 4 years), we found a significant negative association of COMP with medial femorotibial compartment cartilage thickness $(-0.010(-0.019,-0.000) p=0.045)$, and MMP-3 with patellar cartilage thickness $(-9.075(-16.344,-1.807) \mathrm{p}=0.015)$ and total bone area $(-0.047$ $(-0.086,-0.007) p=0.020)$. No significant association was observed between HA and MRI markers.

In the multivariable model for association of MRI-based imaging biomarkers with biochemical markers (assessed after 6-9 years), a significant negative association of total cartilage volume $(-0.0005(-0.0008,-0.0002) p=0.001)$ and total cartilage thickness $(-0.628(-1.143,-0.114) \mathrm{p}=0.017)$ with MMP-3, and total bone area with COMP $(0.270(-0.474,-0.006) p=0.010)$ was observed. No significant association was observed between MRI-based imaging biomarkers and $\mathrm{HA}$.

Conclusion: COMP and MMP-3 levels were negatively associated with knee cartilage thickness assessed 4-years later. Similarly, knee cartilage thickness and volume were negatively associated with COMP and MMP-3 levels assessed 6-9 years later in population-based middle-aged adults, indicating an interdependent negative association of OA-related biochemical markers and MRI-based imaging biomarkers. These results suggest that OA-related biochemical markers may predict future MRI-based imaging biomarkers in middle-aged adults and thus represent possible at-risk populations to target for structure modification interventions.

Acknowledgements: This project was funded by the National Health and Medical Research Council of Australia Project Grant and Royal Hobart Hospital Research Foundation Project Grant.

Disclosure of Interests: Benny Antony Grant/research support from: Investigator-Initiated Clinical Trial support from Nat Rem Ltd., Ambrish Singh: None declared, Leigh Blizzard: None declared, Alison Venn: None declared, Graeme Jones Speakers bureau: Speaker for various pharma, Grant/research support from: Investigator-Initiated Clinical Trial support, John Burgess: None declared, Venkat Parameswaran: None declared, Flavia Cicuttini: None declared, Lyn March: None declared, Changhai Ding: None declared

DOI: 10.1136/annrheumdis-2021-eular.3418

\section{POS0130 ASSOCIATION BETWEEN HYPERTENSION AND OSTEOARTHRITIS}

E. Taskina ${ }^{1}$, E. Strebkova ${ }^{1}$, L. Alekseeva ${ }^{1,2}$, N. Kashevarova ${ }^{1}$, K. Telyshev ${ }^{1}$, S. Anikin ${ }^{1}$, E. Sharapova ${ }^{1}$, D. Kudinsky ${ }^{1}$, A. Lila ${ }^{2,3} .{ }^{1}$ V. A. Nasonova Research Institute of Rheumatology, Osteoarthritis, Moscow, Russian Federation; ${ }^{2}$ Russian Medical Academy of Continuous Professional Education, Rheumatology, Moscow, Russian Federation; ${ }^{3}$ V. A. Nasonova Research Institute of Rheumatology, Director, Moscow, Russian Federation

Background:

Objectives: To evaluate the relationship between arterial hypertension $(\mathrm{AH})$ and the course of knee osteoarthritis $(\mathrm{OA})$. 